\title{
Electrogastrogram Recovery for Correction of Gastric Disorders Using Gastric Stimulator
}

\author{
Govindasamy Gopu1, M. S. GeethaDevasena² \\ ${ }^{1}$ Department Biomedical Engineering, Sri Ramakrishna Engineering College, Coimbatore, India \\ ${ }^{2}$ Department Computer Science Engineering, Sri Ramakrishna Engineering College, Coimbatore, India \\ Email: gopu.govindasamy@srec.ac.in,msgeetha@srec.ac.in
}

Received 9 March 2016; accepted 12 April 2016; published 15 April 2016

Copyright (C 2016 by authors and Scientific Research Publishing Inc.

This work is licensed under the Creative Commons Attribution International License (CC BY). http://creativecommons.org/licenses/by/4.0/

(c) (i) 0pen Access

\begin{abstract}
Gastric disorders play an important role in digestive system of a human subject. Gastric disorder is due to the gastric dysmotility due to delayed gastric emptying and other motor dysfunctions like impaired fundic distention. Nowadays invasive technique like laparoscopy procedure, endoscopy procedure and surgery procedure plays a vital role in investigation of gastric disorders. Electrogastrogram is a noninvasive technique for analyzing digestive system disorders. In this proposed work, stimulation is delivered with the electrode package with camera, which travels through an esophagus to reach stomach and stimulate the interstitial cajal cells. Due to this stimulation the stomach starts, its activity a potential is provided that is recorded with electrode setup, which is called electrogastrogram. It is verified with its normal range $3 \mathrm{cpm}$. The stimulation includes pulses mode and continuous mode. Stomach function is recovered and its frequency is measured and the same is compared with normal range value. Depending upon the subject condition, the gastric activity is improved by setting different values of duty cycle. By this procedure the subject avoid painful procedure, stay in hospital, frequently and the cost is minimum.
\end{abstract}

\section{Keywords}

Gastroparesis, Nausea, Vomiting, LabVIEW, Electro GastroGram, Gastric Electrical Stimulation, Duty Cycle, PIC Microcontroller

\section{Introduction}

Digestive system is a group of organs which works together to convert food into energy and provides of nutrients 
to entire body. Food passes through long tube known as alimentary canal. The alimentary canal is made up of oral cavity, pharynx, esophagus, stomach, small intestine, large intestine and also other organs helps in the digestion. The major function of the system is ingestion, secretion, mixing and movement, digestion, absorption, excretion. Any ailments related to digestive system must be corrected. It would be great if the correction is done with minimal pain, maximum relief and at moderate cost. The project is developed to help Subjects with gastric motility disorders, relieve them from nausea, vomiting and other discomforts due to the ailments.

The human gastric pacemaker was localized to an area in the midcorpus along the greater curve. Pace setter potentials were generated regularly by the pacemaker at a mean frequency 3.2 cycles per min and were propagated circumferentially and aborally from the pacemaker, increasing in amplitude and velocity as they approach the pylorus. Many people in the world are suffering from unexplained dysarhythmias such as ulcer, gastroparesis, functional dyspepsia, gastritis, delayed gastric emptying, etc. These subjects show a variation in the number and pattern of the cycles per minute. The gastric stimulator-Innova paces the stomach so that the natural rhythm is achieved. They work like a heart pacemaker, sending electrical signals to the stomach to tell it to contract and process food. The objective of this project is to design stimulators which cause minimum discomfort and to give relief without surgical intervention.

\section{Need for Current Work}

Any Gastric aliments causes vomiting and nausea. The most severe cases of gastro paresis are refractory to drugs. In the 1990's gastric electrical stimulation (GES) was introduced for the treatment of nausea and vomiting in Subjects with diabetic and idiopathic gastroparesis. Study using gastric pacemaker to deliver high energy depolarizing stimuli to the stomach show promise in promoting gastric emptying and reducing symptoms of gastroparesis. The electrodes are usually placed with surgery. Cost of the surgery is $\$ 21,000$. Improvements were made which aided placements through laparoscopy. Now, a new type of gastric stimulator is designed, for insertion into the muscular layer of the stomach under endoscopic guidance. After anchoring the electrode using a multi-bend endoscope, stimulus either pulsed or continuous sequences are delivered depending on the patient's condition in order to make them relieved from the discomfort of the disorder. Permanent gastric pacers face the problem of re-surgery for battery replacement, failure of the device etc. But temporary stimulators could be delivered any time for multiple Subjects, thus cost of the procedure drastically decreases. EGG could be recorded in Subjects indicating proper electrode stimulation and to note the improvements of Gastric Stimulation.

\subsection{Myoelectrical Control of Gastric Motility}

The two types of cells are central in the function of gastric motility namely Smooth muscle cells (SMC) and Interstitial Cells of Cajal (ICC). SMC are characterized by phasic and tonic contractions under control of autonomic and enteric neurons. ICC serves as rhythm generators and interface between nerves and smooth muscle. Via electrical coupling, ICC regulates smooth muscle membrane potential. $\mathrm{Ca}^{2+}$ entry via voltage sensitive $\mathrm{Ca}^{2+}$ channels trigger smooth Muscle contraction. Gastric myoelectrical control activity (the slow wave) is a rhythmic and omnipresent electrical pattern, which occurs at a frequency of three cycles per minute. Gastric slow waves are generated by the ICC and thought to originate at a site along the greater curvature in the proximal to middle corpus [1].

\subsection{Gastric Disorders}

Gastroparesis is a disorder in which the stomach takes too long to empty its contents. Symptoms of gastroparesis are nausea, vomiting, an early feeling of fullness when eating, weight loss, abdominal bloating, abdominal discomfort. These symptoms may be mild or severe, depending on the person. Cyclical vomiting syndrome is characterized by recurring attacks of severe vomiting, sometimes at regular intervals. The attacks may last for up to 10 days. Due to this the subject may have Headache, abdominal pain, fever may accompany vomiting and eating disorders. Apart from classical symptoms of abdominal pain, diarrhea and/or constipation, about $20 \%$ of the Subjects with IBS also suffer from nausea.

\subsection{Adverse Effects of Drugs}

Beta blockers reduce heart rate; reduce blood pressure, constrict air passages by stimulating the muscles that 
surround the air passages to contract. Cisapride even fatal heart problems have occurred when cisapride was used alone or with other drugs. Diazepam, if taken more frequently or use it for a longer period of, this drug can be habit-forming. The dose may need to be gradually decreased. Severe side effects of Narcan include agitation, hypo and hypertension, cardiac arrhythmias, dyspnea, pulmonary edema, encephalopathy, seizures, coma, and death. Domperidone should not be used in tumor of the pituitary gland or stomach or even for duodenal ulcer because it causes frequent severe stomach cramps, a blockage, tear or perforated ulcer in your intestines, black, tarry bowel motions (stools) or notice blood in your bowel motions, severe liver and kidney disease, abdominal pain, back, leg, or stomach pain, bleeding or crusting sores on lips blistering, burning, redness, scaling, or tenderness of skin blisters on palms of hands and soles of feet, changes in vision or blurred vision, confusion, coughing or difficulty in swallowing, dark-colored urine, dizziness, fainting, fast, pounding, or irregular heartbeat, painful glands, troubled breathing and unusual bleeding or bruising [2]-[5].

\section{Methodology}

\subsection{Proposed Plan-Internal Stimulator}

A seed project was done using LabVIEW proposing the working of Gastric stimulator-Innova. Figure 1 shows the Delivery mode page where the actual values are to be set by the physicist. Once the port is ready, it shoots the stimulus to the patient. The parameter for the stimulus is set by the doctor depending on the subject's severity. The first waveform indicates the stimulus depending on the parameters entered. The later shows the EGG results in cycles per minutes [6].

The main project is developed from the seed project and the step by step procedure to be followed is simply depicted using a flowchart as shown in Figure 2.

- Assess the severity of the disorder.

- The disorder is refractive to drug.

- Try the effects of Gastric Stimulator.

- Choose from variety of modes

- Monitor the progress using EGG.

- Adjust the modes till the patient feels relief.

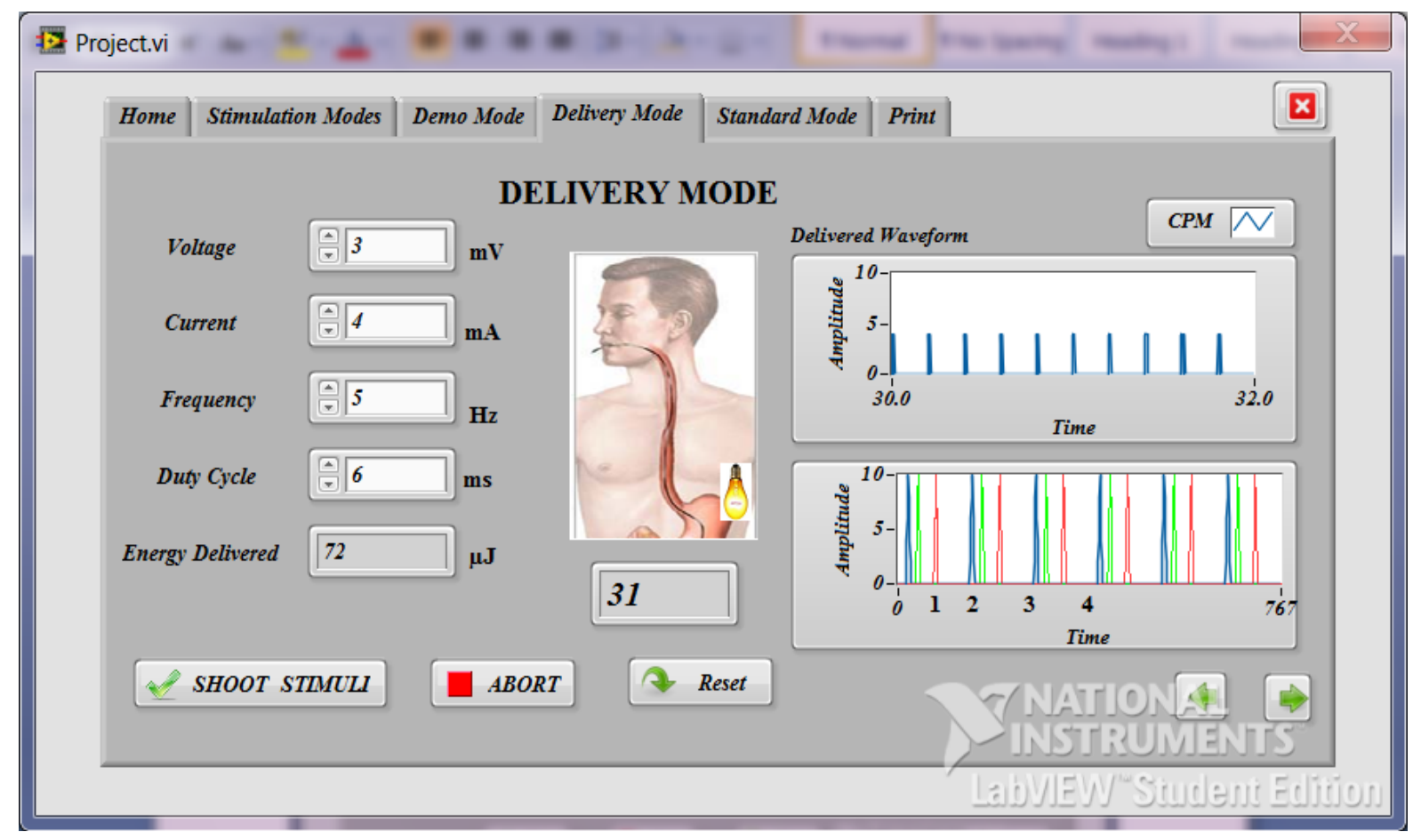

Figure 1. Delivery mode of proposed gastric stimulator. 


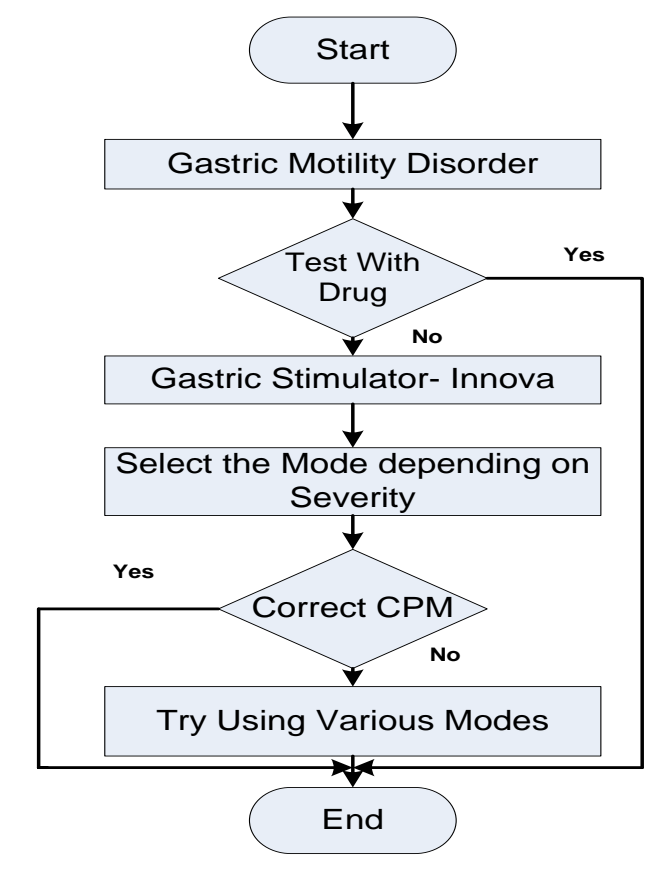

Figure 2. Flow sequence of the procedure.

\subsection{Hardware Setup}

The Block diagram for this Stimulator is shown in Figure 3. The module has a transformer, which steps down the $230 \mathrm{~V}$ to useable level. The next component is the voltage regulator. It provides $5 \mathrm{~V}$ and $12 \mathrm{~V}$ output power supply, which help to operate the total circuitry. It plays a great role in this system because voltage fluctuation may cause harmful to the system. Figure 4 shows the overall hardware set up. Most important component of all the hardware is PIC16F877A microcontroller [7]. It is called the heart of this system. It almost controls the entire component those use in this system.

Firstly it is used to set the PWM, then by using its ADC module it converts its equivalent digital data to the respective output port. According to program it transfers data to different system components. LCD shows visually the current stimulation mode under use. Here $16 \times 2$ LCD modules are used to display the data string. This system also includes another important hardware component which is known as DC motor driver (L298). On connecting the stimulator tip directly to the PIC microcontroller, back E.M.F may damage the microcontroller, so it is very essential. Final component or output component used in this system is the stimulating or the pulse delivery lead tip. Applying desired PWM duty cycle provided by the microcontroller and controlled by the LabVIEW software is applied to the ICC cells to stimulate the stomach muscle. The lead tip is coated with platinum nanoparticles by inter-particle linking using biotin-avidin to render the biocompatibility nature.

\subsection{Software Description}

LabVIEW system design software is at the center of the National Instruments platform. It integrates graphical, text-based, and other programming approaches within a single environment to efficiently create custom software solutions. NI-VISA is the National Instruments implementation of the VISA I/O standard. The points to be noted while programming are given below.

Configure the serial port, then read and write many times in a loop, followed by closing the port after all communication is finished Only the frames of the sequence structure containing the waits are required. Energy delivered for every stimulus is given by multiplying the current, voltage and time. Thus, the work done per unit time in this case is in the order of few micro joules. This implies the patient just feels some signal is passing through his body. This implies the procedure is not painful. Low-frequency/high-energy GES, known as gastric electrical pacing, is able to induce a regular rhythm, restore efficient contractions. 


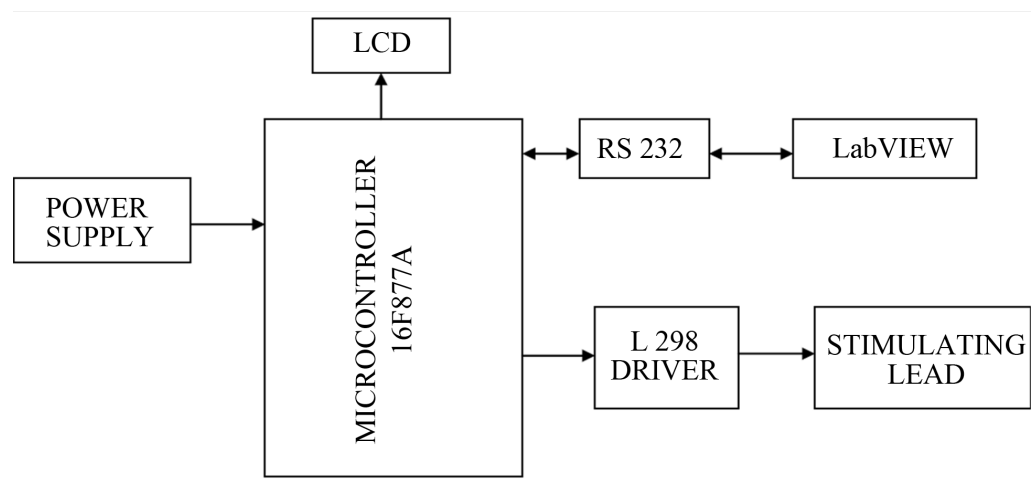

Figure 3. Flow sequence of the procedure.

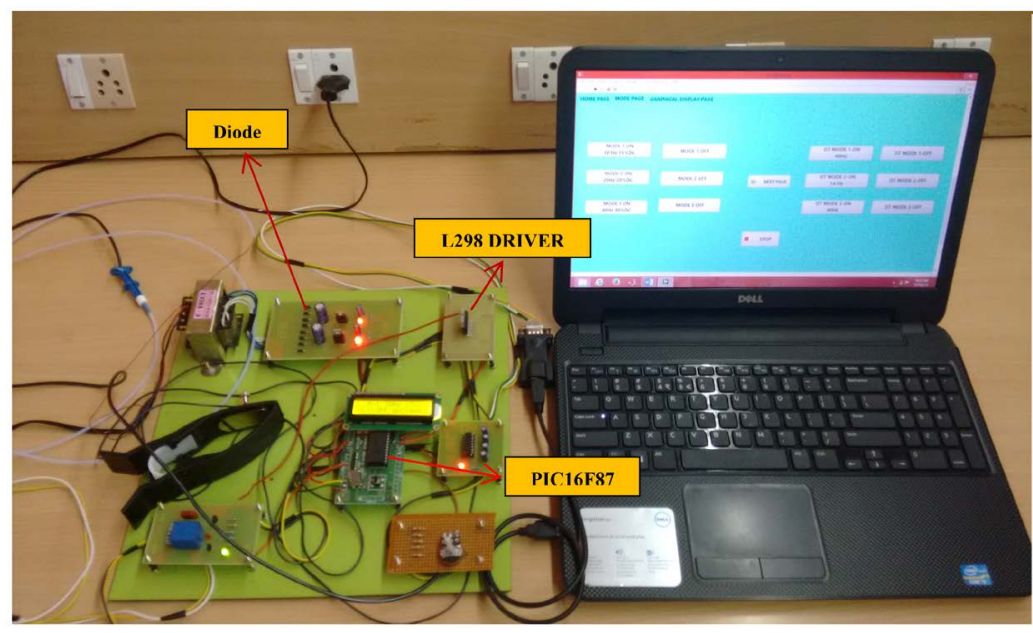

Figure 4. Overall hardware setup.

\subsection{Procedure}

Select the serial port that the device is connected. The status of the device port to receive the pulse is indicated click the proceed button to go next stage. Depending upon the patient condition the required mode is selected from the standard mode by the physicist. Figure 5 and Figure 6 show the home page and Mode page of the stimulator [8] [9]. Once the port is ready, it shoots the stimulus to the patient. Table 1 shows the stimulation parameters considered for continuous and pulsed mode stimulation with this stimulator. For different duty cycle for the both mode the recovered Electro Gastro Gram is noted in $\mathrm{Hz}$.

The EGG signal is acquired from the subject along with noise is generated and the waveform thus obtained is abnormal EGG waveform (below 3 cpm) [10] [11] shown in Figure 7. Once the pulse from the internal stimulator is reached to the patient stomach, the abnormal EGG pattern of the stomach is recovered back to normal function $(3 \mathrm{cpm})$ due to the internal stimulus given as shown in Figure 8.

\section{Conclusions}

Gastro paresis symptoms like nausea, vomiting, and regurgitation, may delay drug absorption. Eventually as the disease severe these diseases become refractive to drugs. Under the endoscopic physician guidance the temporary electrodes for GES can easily be anchored percutaneous. As a result many subjects respond to Gastric Electrical stimulation with reduction of nausea and vomiting. Also the subjects with chronic intestinal pseudo-obstruction, post-surgical gastro paresis and Subjects with severe nausea but normal gastric emptying can also benefit from treatment with GES. GES significantly reduced severity and recover the frequency near to normal for digestive disorders. It is also observed the duty cycle for pules varies for subject to subject and same is fixed with some trial and error method. The duty cycle ratio plays major role in this recovery procedure for gastric 


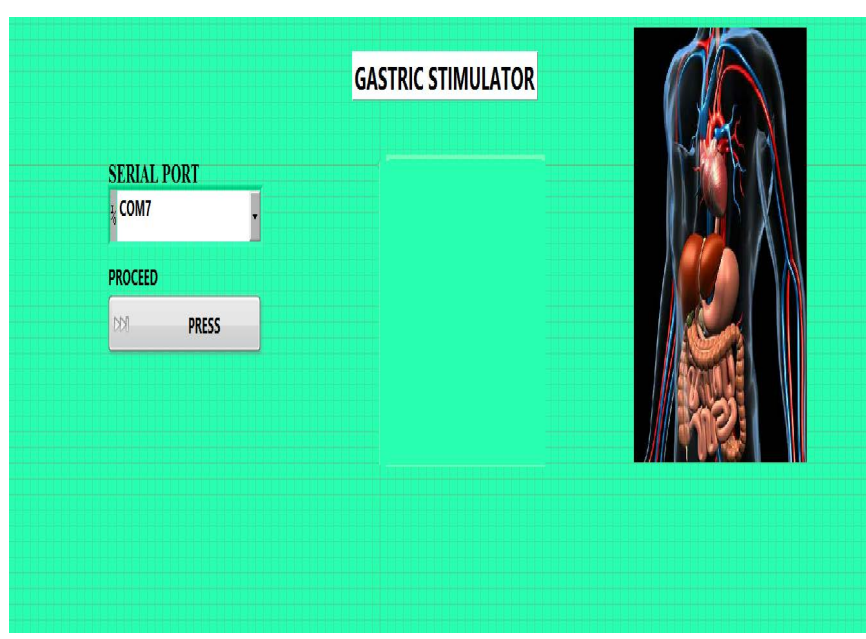

Figure 5. Home page of gastric stimulator-innova.

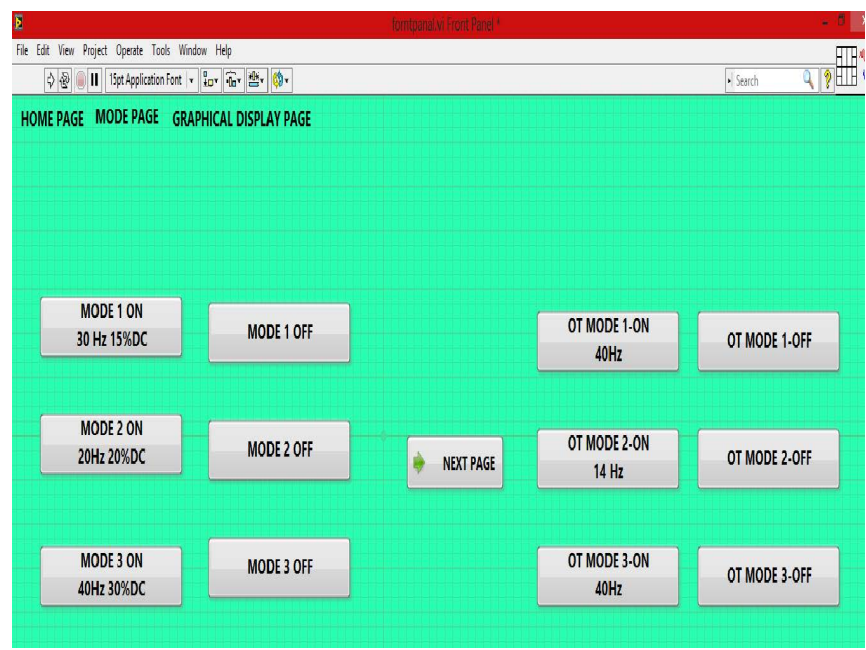

Figure 6. Mode page of gastric stimulator-innova.

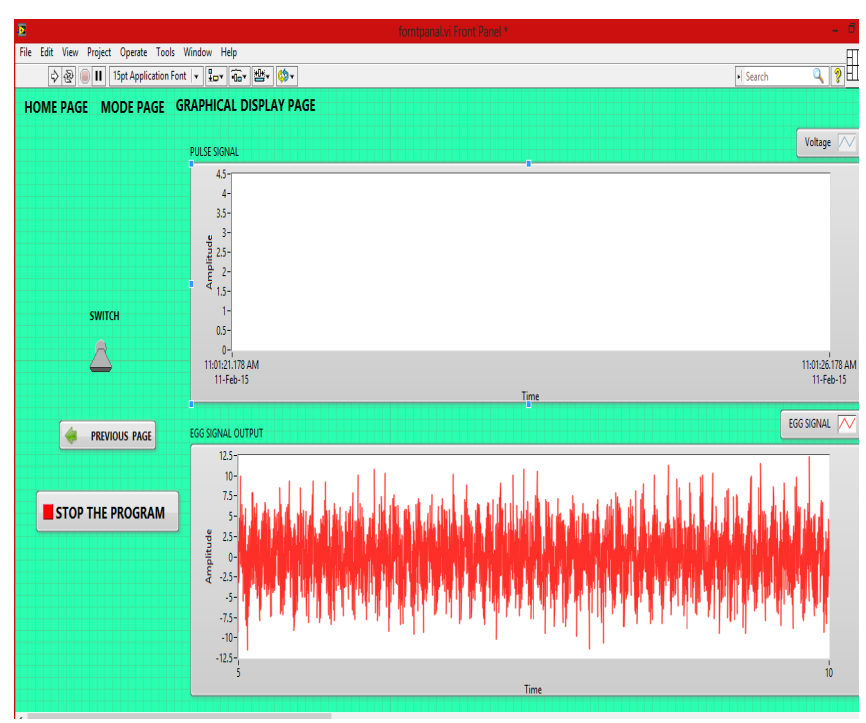

Figure 7. Abnormal EGG waveform. 


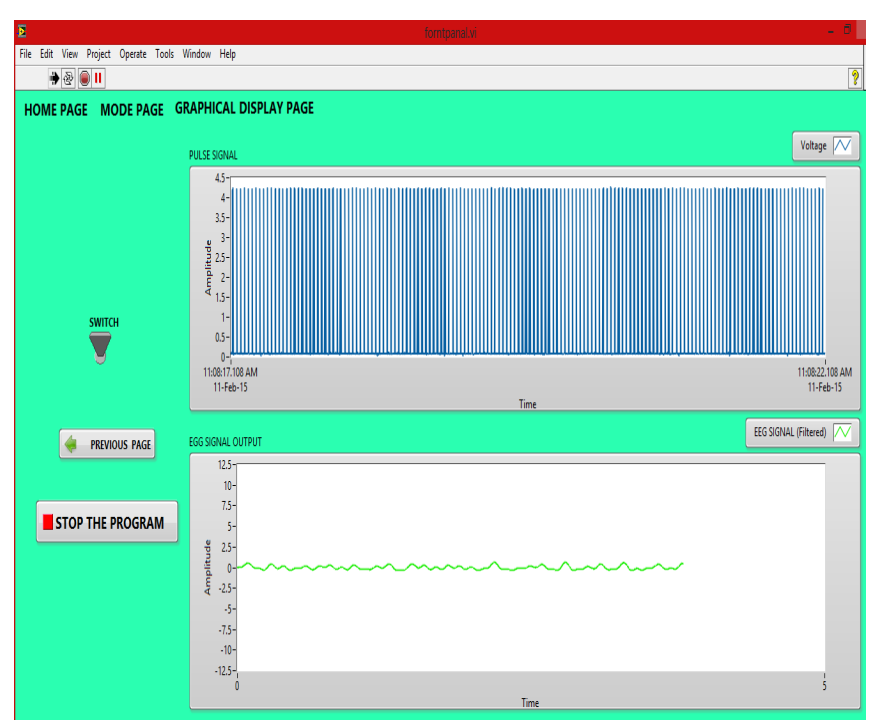

Figure 8. Recovered EGG signal due to stimulation.

Table 1. Pulse specifications.

\begin{tabular}{|cccccc|}
\hline & \multicolumn{2}{c}{ PULSE MODE } \\
\hline $\begin{array}{c}\text { Voltage } \\
\text { (Volts) }\end{array}$ & $\begin{array}{c}\text { Current } \\
\text { (mA) }\end{array}$ & $\begin{array}{c}\text { Duty } \\
\text { Cycle }\end{array}$ & $\begin{array}{c}\text { Frequency } \\
\text { Hz }\end{array}$ & $\begin{array}{c}\text { ON } \\
\text { Time }\end{array}$ & $\begin{array}{c}\text { OFF } \\
\text { Time }\end{array}$ \\
\hline $\mathbf{1 . 8}$ & 6 & $50 \%$ & 40 & 2 & 3 \\
1.8 & 6 & $50 \%$ & 14 & 0.1 & 3 \\
1.8 & 6 & $8 \%$ & 40 & 2 & 3 \\
\hline
\end{tabular}

\begin{tabular}{cccc|}
\hline & \multicolumn{2}{c|}{ CONTINIOUS MODE } & \\
\hline Voltage (Volts) & Current $(\mathbf{m A})$ & Duty Cycle & Frequency Hz \\
\hline $\mathbf{2 . 0}$ & 7 & $15 \%$ & 40 \\
1.5 & 5 & $20 \%$ & 14 \\
1.0 & 3 & $30 \%$ & 40 \\
\hline
\end{tabular}

disorder. This stimulator works efficiently for different mode of operation.

Observing the responder's results and non-responders category, enhancements can be made by adding the number of stimulation modes. Thus effects can be made to optimum level even without using a specialized endoscope. This therapeutic method can be explored further for the treatment of severe conditions of gastric disorders.

\section{Acknowledgements}

Authors thank the management, director and principal of SREC for their motivation in doing this project. The authors also thank the faculty of department of Biomedical engineering for their support and guidance. The authors also thank the gastroenterologist from Sri Ramakrishna Hospital for their support in collecting EGG samples under their monitoring.

\section{References}

[1] Gopu, G., Das, A., Subamathi, T. and Treisa, T.G. (2014) Gastric Stimulator-Innova. I4C2014, MSRIT, Bangalore, 21-22.

[2] Loo, F.D., Palmer, W., Soergel, K.H., Kalbfleish, J.H. and Wood, C.M. (1984) Gastric Emptying in the Patients with 
Diabetes Mellitus. Gastroenterology, 194, 485-494.

[3] Vaisman, N., Weintrob, N., Blumental, A., Yosefsberg, Z. and Vardi, P. (1999) Gastric Emptying in Patients with Type 1 Diabetes Mellitus. Annals of the New York Academy of Sciences, 873, 506-511. http://dx.doi.org/10.1111/j.1749-6632.1999.tb09499.x

[4] Annese,V., Bassotti, G., Caruso, N., et al. (1999) Gastrointestinal Motor Dysfunction, Symptoms and Neuropathy in Non-Insulin Dependent (Type 2) Diabetes Mellitus. J.Clin. Gastroenterol, 29, 171-177. http://dx.doi.org/10.1097/00004836-199909000-00014

[5] Kong, M.F., Horowitz, M., Jones, K.L., Wisharth J.M. and Harding, P.E. (1999) Natural History of Diabetic Gastroparesis. Diabetes Care, 22, 503-507. http://dx.doi.org/10.2337/diacare.22.3.503

[6] Gopu, G., Neelaveni, R. and Porkumaran, K. (2010) An Enhanced Technique for Recording and Analysis of Electrogastrogram Using Active Electrodes. Sri Lanka Journal of Bio-Medical Informatics, 1, 20-26. http://dx.doi.org/10.4038/sljbmi.v1i1.1482

[7] Mazidi, M.A., Kinlay, R.M. and Causey, D. (2008) PIC Microcontroller. Prentice Hall Inc., Upper Saddle River.

[8] Gopi, M. and Gopu, G. (2015) Closed Loop Analysis of Bridgeless SEPIC Converter for Drive Applications. International Journal of Power Electronics, 6, 253-259.

[9] Gopi, M. and Gopu, G. (2015) Closed Loop Analysis of Single-Phase Soft-Switching PFC Converter. Middle-East Journal of Scientific Research, 23, 66-70.

[10] Gopu, G., Neelaveni, R. and Porkumaran, K. (2008) Investigation of Digestive System Disorders Using Electrogastrogram. Proceedings of IEEE International Conference on Computer and Communication Engineering (ICCCE'08), Volume 2, Kuala Lumpur, 13-15 May 2008, 201-205.

[11] Gopu, G., Neelaveni, R. and Porkumaran, K. (2009) Noninvasive Technique for Acquiring and Analysis of Electrogastrogram. IJCA, Special Issue on CASCT, 2, 73-76. 